\title{
An infinitary paradox of denotation
}

\section{GABRIEL UZQUiano}

A well-known paradox due to G. G. Berry ${ }^{1}$ illustrates the fact that we encounter paradoxes when we inquire into the denotations of definite descriptions. Berry's paradox arises when one considers the definite description:

The least integer not nameable in fewer than nineteen syllables.

Since there is a finite number of English definite descriptions containing fewer than nineteen syllables, there are (positive) integers not nameable by

${ }^{1}$ Berry presented the paradox in a letter to Russell in 1904. The paradox is reported in page 61 of Whitehead and Russell 1910. 
any definite description containing fewer than nineteen syllables. But it would seem that the definite description 'the least integer not nameable in fewer than nineteen syllables' succeeds in naming the least such integer in fewer than nineteen syllables, which is absurd.

This and related paradoxes by Julius König and Jules Richard, both discovered in 1905, make plain that our naive conception of denotation is just as afflicted with inconsistencies as our naive conceptions of truth and satisfaction. The paradoxes of denotation, however, have attracted considerably less attention that the paradoxes of truth and satisfaction.

One question that has been much discussed lately is whether the paradoxes of truth and satisfaction turn on self-reference. In Yablo (1993) Stephen Yablo, a seemingly non-self-referential paradox of truth, which may be generalized to apply to satisfaction. However, Yablo's example has been recently challenged in Priest (1997) and Beall (2001). A defence of Yablo's example in the face of some of the objections raised by Priest (1997) is due to Sorensen (1998). Thus the issue of whether Yablo's paradox avoids self-reference remains highly controversial. What is beyond question is that Yablo's example is genuinely paradoxical and that it demonstrates a new variety of the richness of the family of paradoxes of truth and satisfaction.

But what is the situation for the paradoxes of denotation? A standard view is that Berry's paradox and the other paradoxes of denotation turn on self-reference. Berry's paradox certainly trades on self-reference, since the definite description used to generate the contradiction implicitly quantifies over all English phrases, including itself. A cursory look at the paradoxes of König and Richard will reveal similar uses of self-reference. The purpose of this note, however, is to describe a paradox of denotation that avoids self-reference, if Yablo's paradox does.

Imagine an infinite queue of people tailed back at the gates of Hilbert's hotel. ${ }^{2}$ While they wait, they are all asked to please choose a natural number that will determine their room number. In response to this request, each person in the queue sets out to choose the least number not chosen by anybody standing behind her in the queue. To that purpose, for every $n$, the $n$th member of the queue uses a definite description of the form:

$\left(\mathrm{D}_{n}\right)$ The least natural number not denoted by any $\mathrm{D}_{k}$ for $k>n$.

As a result, we obtain an infinite list of definite descriptions:

$\left(\mathrm{D}_{1}\right)$ The least natural number not denoted by any $\mathrm{D}_{k}$ for $k>1$,

$\left(\mathrm{D}_{2}\right)$ The least natural number not denoted by any $\mathrm{D}_{k}$ for $k>2$,

$\left(\mathrm{D}_{3}\right)$ The least natural number not denoted by any $\mathrm{D}_{k}$ for $k>3$,

which, again, has as its $n$th element:

${ }^{2}$ I borrow the fantasy of an infinite queue from Sorensen (1998). 
$\left(\mathrm{D}_{n}\right)$ The least natural number not denoted by any $\mathrm{D}_{k}$ for $k>n$.

Notice that no use of self-reference or circularity seems necessary to specify the sequence. Each definite description involves reference exclusively to definite descriptions further down the list. Unfortunately, plausible as it may seem, this claim is not beyond doubt. If some of the arguments deployed in Priest 1997 and Beall 2001 for the claim that Yablo's paradox turns on a subtle form of self-reference are successful, then it will not be difficult to generalize them to apply to the present case. When suitably generalized, they will suggest that our specification of the infinite descending sequence of definite descriptions is subtly circular, and that this circularity is to blame for the paradox that will ultimately ensue. ${ }^{3}$

But let me return to our scenario. It would seem that each definite description in our list should either denote a natural number or fail to denote. But now; did all of the persons in the queue manage to choose a room number? It seems not all did. For not all the definite descriptions in the list denote a natural number. If they all did, then each $\mathrm{D}_{n}$ would denote a number strictly greater than the denotation of any description further down the list. That is, each $\mathrm{D}_{n}$ would denote a number strictly greater than the denotation of any $\mathrm{D}_{k}$ for $k>n$. But while no nutural number has an infinite number of predecessors, there is an infinite number of phrases after each $\mathrm{D}_{n}$. Thus we infer:

(i) Not all definite descriptions in the list denote a natural number.

And yet, it seems that all of the members of the queue should have been able to choose a natural number. For take some definite description in the list such as, for example, $\mathrm{D}_{n}$. Either (a) there is no $k>n$ such that $\mathrm{D}_{k}$ denotes a natural number or (b) there is some $k>n$ such that $\mathrm{D}_{k}$ denotes a natural number. If (a), then 0 is the least number not denoted by any $\mathrm{D}_{k}$ for $k>n$. Therefore, $\mathrm{D}_{n}$ denotes 0 . If (b), then there must be a least $k>n$ such that $\mathrm{D}_{k}$ denotes a natural number. Let $l$ be such a number and let $m$ be the natural number denoted by $\mathrm{D}_{l}$. If $\mathrm{D}_{l}$ denotes $m$, then no definite description following $\mathrm{D}_{l}$ in the list denotes $m+1$ (since obviously $m+1>m$ ). But then, $m+1$ is the least natural number not denoted by any $\mathrm{D}_{k}$ for $k>n$. Thus $\mathrm{D}_{n}$ denotes $m+1$. In either case, (a) or (b), $\mathrm{D}_{n}$ denotes a natural number. We conclude:

(ii) All definite descriptions in the list denote a natural number.

From (i) and (ii), we obtain a contradiction.

If Yablo's paradox is genuinely non-self-referential, then self-reference is not necessary for the formulation of the paradoxes of truth and satisfac-

3 The suggestion is that the explicit formulation of the sequence requires each definite description $\mathrm{D}_{n}$ to use its own location in the sequence as a reference point to specify the range of definite descriptions used to determine its referent, i.e. the definite descriptions after $\mathrm{D}_{n}$. 
tion. But the paradox of denotation I have just given doesn't involve selfreference, if Yablo's paradox doesn't. I conclude that if Yablo's paradox is non-self-referential, then self-reference isn't necessary for the formulation of paradoxes of denotation either. If, despite all appearances to the contrary, self-reference is subtly involved in the paradox I have just given, then I trust I will at least have hinted that the family of paradoxes of denotation is just as rich and varied as the paradoxes of truth and satisfaction. ${ }^{4}$

\author{
University of Rochester \\ Rochester, NY 14627-0078, USA \\ gabriel.uzquiano@rochester.edu
}

\title{
References
}

Beall, Jc. 2001. Is Yablo's paradox non-circular? Analysis 63: 176-87.

Priest, G. 1997. Yablo's paradox. Analysis 57: 236-42.

Sorensen, R. 1998. Yablo's paradox and kindred infinite liars. Mind 107: 137-55.

Whitehead, A. N. and B. Russell. 1910. Principia Mathematica. Cambridge: Cambridge

University Press. Page references are to the second edition, published in 1927.

Yablo, S. 1993. Paradox without self-reference. Analysis 53: 251-52.

${ }^{4}$ Thanks to Michael Clark, Agustín Rayo, Keith Simmons and Steve Yablo. 